\title{
Effects of a Distribution on Gap Acceptance Functions on Pedestrian Queues
}

\author{
George H. Weiss
}

(November 8, 1963)

\begin{abstract}
The theory of pedestrian queueing has been developed by several authors under the assumption that all pedestrians have the same gap acceptance functions. In this paper the theory is modified to take into account a distribution of gap acceptance functions. Some calculations made with the new formulas seem to indicate that only if traffic is very heavy will the difference between the results of the two theories become noticeable.
\end{abstract}

The theory of pedestrian queueing at a traffic intersection has been developed by several authors, [1-3]. ${ }^{1}$ In this theory it is assumed that pedestrians arrive at a street corner according to a Poisson process with parameter $\lambda$. They observe the arrival of cars in a single lane at times $t_{1}, t_{2}, \ldots$ such that the gaps $t_{i}-t_{i-1}$ are identically distributed random variables with probability density functions $\phi(t)$. Each pedestrian decides to cross or not according to a gap acceptance function $\alpha(t)$ which is defined to be the probability that a pedestrian will choose to cross the street if the gap (measured in time) to the next arriving car is $t$. In addition the assumption is made that each pedestrian makes a decision to cross when he first arrives at the street corner. If he chooses not to cross at his time of arrival he joins the group at the intersection and thereafter does not cross independently of the group. The group makes a decision, at the time of arrival of each car, whether or not the succeeding gap is large enough to permit it to cross. The assumption has been made that the gap acceptance function of the group is $\alpha(t)$, the same as for the individual.

There are several unrealistic assumptions in this theory, as was pointed out in [3]. One of the chief unrealistic features of the theory is that the group of pedestrians crosses as a whole, so that there is no latitude to describe differences in decision procedures. It is the purpose of this paper to calculate the effects of variation in gap acceptance functions in a population, on the theory previously developed. We shall see that unless the variation in gap acceptance functions is very great, the assumption of a single gap acceptance function is not a bad one.

Let us first assume that there are a finite number of gap acceptance functions $\alpha_{1}(t), \alpha_{2}(t), \ldots \alpha_{k}(t)$ and that people with $\alpha_{i}(t)$ arrive at the corner in accordance with a Poisson process with rate parameter $\lambda_{i}$. It is also assumed that pedestrians in each group arrive and depart independently of those in any other group.
We shall study the embedded Markov chain of the number of pedestrians in queue each time a car on the road passes the group of people. There are two state probability vectors which are relevant in the present problem. These are

$$
\begin{aligned}
\boldsymbol{\theta}(i) & =\left(\theta_{0}(i), \theta_{1}(i), \ldots\right) \\
\boldsymbol{\theta} & =\left(\theta_{0}, \theta_{1}, \theta_{2}, \ldots\right)
\end{aligned}
$$

where $\theta_{j}(i)$ is the probability that there are $j$ pedestrians of group $i$ in queue, and $\theta_{j}$ is the probability that the total number of pedestrians in queue is $j$. That is to say, if $N_{r}$ is the number of pedestrians of the $r$ th type in queue at a regeneration point when the queue is in an equilibrium state ${ }^{2}$ then

$$
\begin{aligned}
\theta_{j}(i) & =\operatorname{Pr}\left\{N_{i}=j\right\} \\
\theta_{j} & =\operatorname{Pr}\left\{N_{1}+N_{2}+\ldots+N_{k}=j\right\} .
\end{aligned}
$$

The generating function for the $\theta_{j}(i)$ has been derived in [3]. It is

$$
\psi_{i}(s)=\sum_{j=0}^{\infty} \theta_{j}(i) s^{j}=\frac{\Delta_{i}(s)}{1-\epsilon_{i}(s)}
$$

where

$$
\begin{aligned}
& \Delta_{i}(s)=\int_{0}^{\infty} \alpha_{i}(t) \phi(t) e^{-\lambda T_{i}(t)(1-s)} d t \\
& \epsilon_{i}(s)=\int_{0}^{\infty}\left(1-\alpha_{i}(t)\right) \phi(t) e^{-\lambda T_{i}(t)(1-s)} d t
\end{aligned}
$$

and

$$
T_{i}(t)=\int_{0}^{t}\left(1-\alpha_{i}(u)\right) d u .
$$

Therefore, by the assumption of independence, the 
generating function for the $\theta_{j}$ can be written

$$
\psi(s)=\sum_{j=0}^{\infty} \theta_{j} s^{j}=\psi_{1}(s) \psi_{2}(s) \ldots \psi_{k}(s) .
$$

The present theory can be extended to the case where gap acceptance functions depend on a continuous parameter $\nu, \alpha(\nu, t)$. Let the parameter $\nu$ have a density function $g(\nu)$, and let the Poisson rates also be dependent on $\nu$. Then if $\psi(\nu, s)$ replaces $\psi_{i}(s)$ in eq (6) we may write as the generalization of the last equation

$$
\log \psi(s)=\int \log \psi(\nu, s) g(\nu) d v
$$

where integration is over the range of $\nu$. Other formulas in [3] can be similarly generalized.

Let us examine some particular results for the present more general theory. It is shown in [1] that if $\psi(t)$ has the form $\phi(t)=\sigma \exp (-\sigma t)$ and $\alpha(t)$ is a step function

$$
\begin{aligned}
\alpha(t) & =0 t \leqslant T \\
& =1 t>T
\end{aligned}
$$

then the expected number of pedestrians in queue is

$$
\mu=\frac{\lambda}{\sigma}\left(e^{\sigma T}-1\right) .
$$

Now let $\lambda$ and $T$ be functions of $\nu$. The formula for the expected number in queue now becomes

$$
\mu=\int \frac{\lambda(\nu)}{\sigma}\left(e^{\sigma T(\nu)}-1\right) g(\nu) d \nu .
$$

Let us examine the situation in which $\lambda(\nu)=\lambda$, a constant independent of $\nu, T(\nu)$ is the function

$$
T(\nu)=T_{0}(1+\nu) \quad-\frac{\epsilon}{2} \leqslant \nu \leqslant \frac{\epsilon}{2}
$$

and $g(\nu)$ is

$$
\begin{aligned}
g(\nu) & =\frac{1}{\epsilon} \quad-\frac{\epsilon}{2} \leqslant \nu \leqslant \frac{\epsilon}{2} \\
& =0 \text { otherwise. }
\end{aligned}
$$

The integral in eq (10) is elementary and leads to the result

$$
\mu=\frac{\lambda}{\sigma}\left[e^{\sigma T_{0}} \frac{\sinh \left(\frac{\sigma T_{0} \epsilon}{2}\right)}{\sigma T_{0} \epsilon / 2}-1\right] .
$$

If $\epsilon$ is small then

$$
\mu=\frac{\lambda}{\sigma}\left(e^{\sigma T_{0}}-1\right)+e^{\sigma T_{0}} \frac{\lambda \sigma}{24} T_{0}^{2} \epsilon^{2}+O\left(\epsilon^{4}\right) .
$$

A. J. Goldman (private communication) has pointed out that the same sort of expansion can be derived for general $g(\nu)$ symmetric around $\nu=0$.

Supposing now that the parameters have the particular values

$$
T_{0}=5 \mathrm{sec}, \sigma=0.1 \mathrm{sec}^{-1},
$$

the ratio of $\mu / \lambda$ for random gap acceptance functions to that for a single gap acceptance function, calculated from eq (13), is as shown in table 1 for different values of $\epsilon$. Clearly, with these values of the parameters one could not hope to distinguish between the simpler and the more complicated theories experimentally except at high traffic densities. For example if $\sigma$ is increased to $0.5 \mathrm{sec}^{-1}$ and the other parameters held fixed the values of $\mu_{\text {random }} / \mu_{\text {single }}$ are as given in table 2. When $\epsilon$ is as great as 1.2 it might be possible to detect a difference between the two types of theory (when the critical gaps range uniformly between 3.5 and $6.5 \mathrm{sec}$ as contrasted to a single gap acceptance function of $5 \mathrm{sec}$ ) but the average headway between cars of $2 \mathrm{sec}$, or a flow of $1800 \mathrm{cars} / \mathrm{hr}$ is abnormally high. Further detailed calculations of the generaliza-

TABLE 1. Value of $\mu_{\text {random }} / \mu_{\text {single }}$ as a function of $\epsilon$ for $T_{0}=5 \mathrm{sec}, \sigma=\mathbf{0 . 1 ~ \mathrm { sec } ^ { - 1 }}$

\begin{tabular}{c|c|c|c|c|c|c|c|c|c|c|c}
\hline \hline$\epsilon$ & 0 & 0.2 & 0.4 & 0.6 & 0.8 & 1.0 & 1.2 & 1.4 & 1.6 & 1.8 & 2.0 \\
\hline$\frac{\mu_{\text {random }}}{\mu_{\text {single }}}$ & 1.00 & 1.00 & 1.00 & 1.00 & 1.01 & 1.03 & 1.04 & 1.05 & 1.07 & 1.09 & 1.11 \\
\hline
\end{tabular}

TABLE 2. Values of $\mu_{\text {random }} / \mu_{\text {single }}$ as a function of $\epsilon$ for $T_{0}=5 \mathrm{sec}, \sigma=0.5 \mathrm{sec}^{-1}$

\begin{tabular}{c|c|c|c|c|c|c|c|c|c|c|c}
\hline \hline$\epsilon$ & 0 & 0.2 & 0.4 & 0.6 & 0.8 & 1.0 & 1.2 & 1.4 & 1.6 & 1.8 & 2.0 \\
\hline$\frac{\mu_{\text {random }}}{\mu_{\text {single }}}$ & 1.00 & 1.01 & 1.03 & 1.06 & 1.11 & 1.17 & 1.25 & 1.34 & 1.44 & 1.57 & 1.72 \\
\hline
\end{tabular}

${ }^{2}$ It is shown in [3] that such a state always exists except for extremely pathological 
tions of formula in [3] lead also to the conclusion that it would be very difficult to distinguish between a theory which requires a single gap acceptance function for the entire population, and one which allows a distribution of such functions, if one looks at expectation values alone. It is probable that the effects of a distribution of gap acceptance functions are not important except in heavy traffic, when it often happens that several members of a group of pedestrians will cross while others do not.

\section{References}

[1] J. C. Tanner, The delay to pedestrians crossing a road, Biometrika 38, 383 (1951).

[2] A. J. Mayne, Some further results in the theory of pedestrians and road traffic, Biometrika 41, 375 (1954).

[3] G. H. Weiss, An analysis of pedestrian queueing, J. Res. 67B (Math. and Math. Phys.) No. 4, 229-243 (1963).

(Paper 68B1-113) 